\title{
MICROFABRICATION AND THERMAL BEHAVIOR OF MINIATURE CESIUM-VAPOR CELLS FOR ATOMIC CLOCK OPERATIONS
}

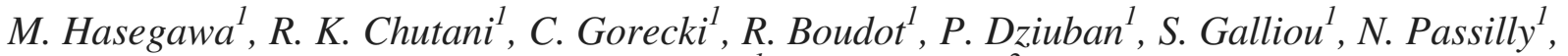 \\ V. Giordano ${ }^{1}$ and A. Jornod ${ }^{2}$ \\ ${ }^{1}$ FEMTO-ST (CNRS UMR 6174/UFC/ENSMM/UTBM), UFR Sciences et techniques, \\ 16 Route de Gray, 25030 Besançon, France \\ ${ }^{2}$ Swatch Group Research and Development Ltd, 2074 Marin, Switzerland
}

\begin{abstract}
We report on the microfabrication and packaging of Cs-vapor cells filled with $\mathrm{Ne}$ or Ar for micro atomic clocks (MACs) based on the coherent population trapping (CPT). We present a two-step anodic bonding process to control the cell atmosphere for long-term operation of MACs. Furthermore, the results of thermal analysis of a cell embedded in the physical package are shown in order to discuss the optimal design of the package for the thermal management of the MAC.
\end{abstract}

\section{INTRODUCTION}

Micro atomic clocks (MACs) employing the principle of coherent population trapping (CPT) have been intensively investigated because of their capability to miniaturize the physics package, which also contribute to the reduction of the power consumption [1-3]. In MACs, CPT resonance is obtained in a micro Cs-vapor cell containing Cs and a buffer gas at a required pressure. The Cs-vapor cell is illuminated by a high-frequency modulated laser beam, which is subsequently collected by a photodiode which delivers a signal to lock the frequency of a local oscillator on the well-defined atomic resonance $(9.192631770 \mathrm{GHz})$.

In this paper, we report the studies on the microfabrication and packaging of Cs-vapor cells for MACs, which were carried out in the frame of the European project "MEMS Atomic Clocks for Timing, Frequency Control \& Communications (MAC-TFC)".

Micro Cs-vapor cells are fabricated by the conventional micromachining techniques, namely, patterning of the through-hole cavities in Si using photolithography and the dry/wet etching, and subsequent cell-sealing by anodic bonding of two glass plates to both surfaces of Si. For the cell-fabrication, inclusion of reactive and volatile Cs in the cell is one of the key technologies. Recently our research group have proposed a simple Cs filling method using the pill-type $\mathrm{Cs}$ dispenser $\left(\mathrm{Cs}_{2} \mathrm{CrO}_{4}\right.$ and $\mathrm{Zr}$ - $\mathrm{Al}$ alloy mixture) developed by SAES Getters [4]. The dispenser is stable up to $500^{\circ} \mathrm{C}$ while it release $\mathrm{Cs}$ vapor when heated at $\sim 700^{\circ} \mathrm{C}$. By incorporating the dispenser in the cell and by locally heating it with a high-power laser source after sealing of the cell, we are able to obtain required $\mathrm{Cs}$ vapor without any damages to $\mathrm{Si}$ and glass as well as the quality of Si-glass bonding [5]. Based on this technique, we carried out further studies to fabricate the micro Cs-vapor cell suitable for MAC-TFC requirements. One of the important requirements for our MAC is to achieved a short-term frequency stability of
$6 \times 10^{-10}$ at $1 \mathrm{~s}$. To reach this objective, the Cs-vapor cells should be filled with a buffer gas (in our case Ne or Ar), which suppress the broadening of the CPT signal caused by wall-collisions of Cs atoms. Therefore, we focused on our attention on the buffer gas filling step and developed a two-step anodic bonding in order to control the cell atmosphere for long-term periods. The cell atmosphere and its aging behavior were characterized by the optical and CPT spectroscopy.

The micro Cs-vapor cell will be embedded in the integrated physics package based on low temperature co-fired ceramics (LTCC) technology, which will achieve the simple and low-cost manufacturing of MACs. Toward the integration of the cell in the physics package, we discuss a concept of the physics package design. We show the results of thermal analysis of the packaged cell to optimize the thermal management of the MAC.

\section{FABRICATION OF MICRO Cs-VAPOR CELLS}

The Cs-vapor cells are made of a $\mathrm{Si}$ wafer sandwiched between two borosilicate glass wafers (Fig. 1). The cell contains two cavities (through-holes): one for CPT resonance detection and the other for storing Cs dispensing. These cavities are connected through micro surface channels to filter the particles from $\mathrm{Cs}$ dispenser during laser activation process. The inner thickness of the cell is $1.4 \mathrm{~mm}$ which will be sufficient for achieving the objective short-term stability, $6 \times 10^{-10}$ at $1 \mathrm{~s}$.

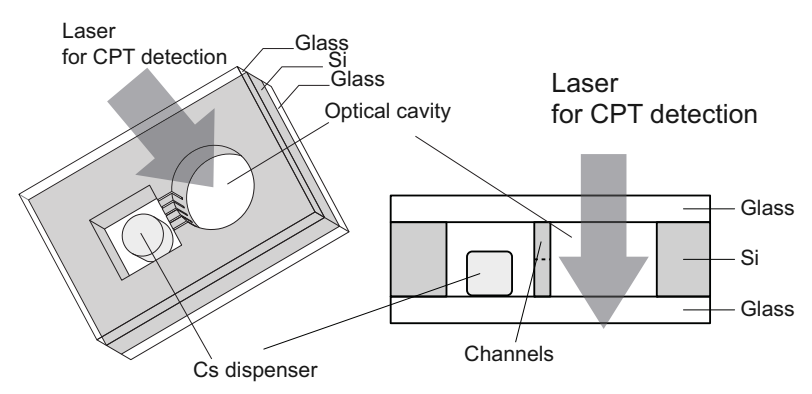

Figure 1: Schematic of Cs-vapor cell.

First, an array of cell cavities and the filtration channels are patterned and etched in Si (Fig. 2, step1). For this step, we employ the deep reactive ion etching (DRIE) because this technique is convenient for precise fabrication of the cell structure. However, through-etching of the 1.4-mm thick Si 
by DRIE is not optimal because the etching quality deteriorates when the etching depth exceeds $500 \mu \mathrm{m}$, mainly because of the "grass" growth. The grass growth was suppressed significantly by the optimization of process parameters (mask material and design, etching temperature, etch-stop layer), which allows us to fabricate the required though-wafer cavities and channels as shown in Fig. 3a. However, after this DRIE process, the etched surfaces near the top and bottom ends of the wafer were covered with the scallop-shaped defects (seen as the white patterns in Fig 3b). These defects are not preferable because they could trap Cs atoms as well as the contaminants which may react with Cs. The defects on the sidewall surface along with the fluorocarbon deposits were effectively removed by immersing the sample in $\mathrm{KOH}$ aqueous solution $(41 \mathrm{wt} \%$, $55^{\circ} \mathrm{C}$ ) for $30 \mathrm{~min}$ (Fig.4). As is seen in Fig. 4b, the scallop-shaped defects were etched completely.

Step 1: Etching of cavities in $\mathrm{Si}$
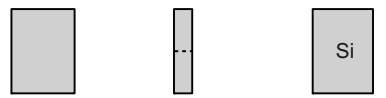

DRIE

$\mathrm{KOH}$ etching

Step 2: Anodic bonding of Si and bottom-glass

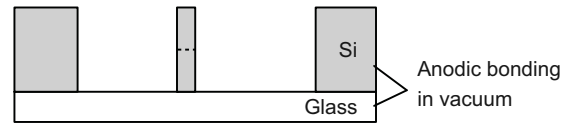

Step 3: Filling of cells with buffer gas

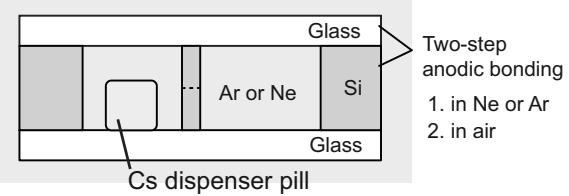

Step 4: Cs dispensing by a dispenser activation Laser

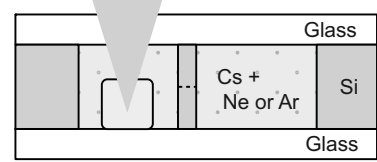

Figure 2: Fabrication steps of micro Cs-vapor cell.
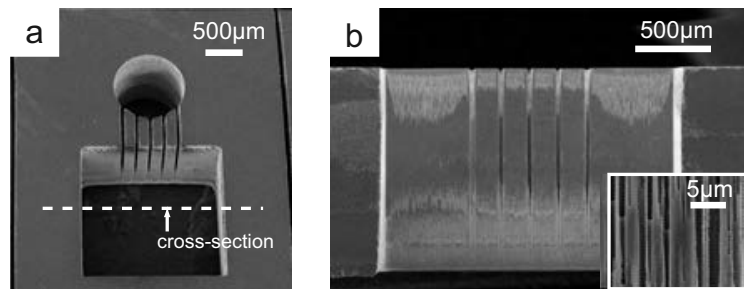

Figure 3: (a) Top-view and (b) cross-section SEM images of cell-cavities fabricated by DRIE (inset: magnified image of the defect-portion).
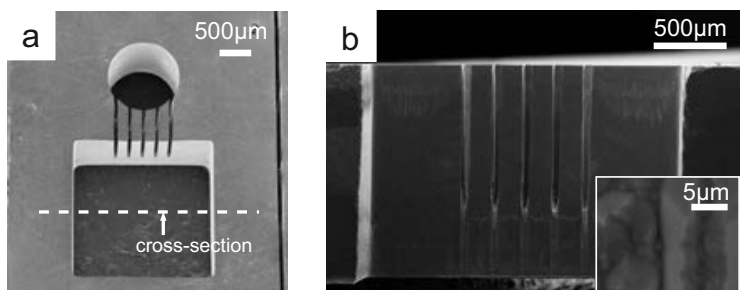

Figure 4: (a) Top-view and (b) cross-section SEM images of cell-cavities after short $\mathrm{KOH}$ etching (inset: magnified image of the defect-portion).

The Si wafer is then anodically bonded to the first glass wafer in vacuum (Fig. 2, step 2). Once a Cs dispenser pill (SAES Getters) is placed in the cavity, the cell is sealed by anodic bonding in the chamber filled with a buffer gas (Ne or Ar) at a required pressure (Fig. 2, step 3). For this step, the electrical breakdown between the metal contact to the cathode electrode and the anode electrode stage was the serious technological issue. For the pressure rage $5-20 \mathrm{kPa}$, which is required for the Cs-vapor cell, the breakdown voltage increases with an increase in pressure according to the Paschen's law [6], and is 250-320 V for $\mathrm{Ne}$ and 365-585 $\mathrm{V}$ for Ar, respectively. The anodic bonding at less than those breakdown voltages resulted in an increase in the defect-area to more than $13 \%$ of the wafer surface. To improve the bonding quality, we applied a two-step anodic bonding process. In this method, the sample is firstly pre-sealed at a voltage lower than the breakdown voltage of the gas atmosphere in the chamber (pre-sealing step). Subsequently, the sample is bonded at a higher voltage $(900 \mathrm{~V})$ in air (final-sealing step). This method successfully improves the bonding quality. The bonding-defect area of the sample after the two-step bonding is less than $6 \%$ of a whole wafer surface, which is equivalent to that of the sample fabricated by standard operation of anodic bonding in vacuum.

After the sealing of the wafer, the array of microcells is diced into single cell chips with the external dimensions of $4 \times 6 \times 2.4 \mathrm{~mm}^{3}$ (Fig. 5). Then, the Cs dispenser pill in the cell is locally heated for $1 \mathrm{~min}$ with a pigtailed diode laser source, operating at a wavelength of $808-\mathrm{nm}(1.3 \mathrm{~W})$ in order to fill the cell with required Cs vapor.

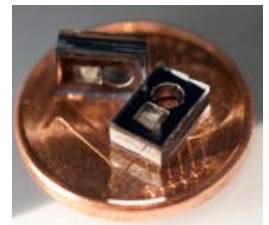

Figure 5: Cs-vapor cells on a 1-cent euro coin.

\section{SPECTROSCOPIC STUDY AND AGING TESTS OF CS-VAPOR CELLS}

The Cs-vapor cells were characterized by optical absorption spectroscopy to confirm the presence of Cs vapor and a buffer gas. The curves in Figs. 6 compares the optical absorption spectrum (D2 transition) obtained from a micro 
Cs-vapor cell filled with $\mathrm{Ne}$ (expected pressure of $10 \mathrm{kPa}$ ) with the spectrum obtained from a cm-scale $\mathrm{Cs}$ reference cells (without buffer gas). For the micro Cs-vapor cell filled with $\mathrm{Ne}$, the two absorption peaks of D2 transition are shifted and broadened compared to those of the Cs reference cell, demonstrating the presence of the buffer gas. Employing the optical red shift coefficient of $-4.25 \mathrm{MHz} / \mathrm{kPa}$ for $\mathrm{Ne}$ [7], the buffer gas pressure in this cell is estimated to be $9.8 \pm 0.09 \mathrm{kPa}$. This result essentially corresponds to the expected pressure $(10 \mathrm{kPa})$.

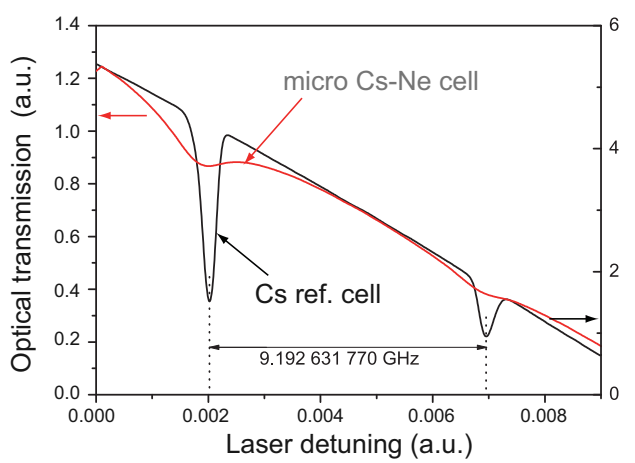

Figure 6: Optical absorption spectroscopy of micro Cs-vapor cell filled with $\mathrm{Ne}$. The expected pressure is $10 \mathrm{kPa}$. The optical absorption measurement was carried out at $80^{\circ} \mathrm{C}$.

The micro Cs vapor-cells were further characterized by CPT spectroscopy employing a laboratory-prototype Cs CPT-clock setup. Figure 7 shows the CPT resonance (D2 transition) obtained in the cell filled with $\mathrm{Ne}(8.1 \mathrm{kPa})$. The linewidth of the CPT signal is $2.52 \mathrm{kHz}$, which is much smaller than that of the similar micro Cs-vapor cell without a buffer gas (line width, $270 \mathrm{kHz}$ ), showing the narrowing effect of the buffer gas. Furthermore, the clock frequency stability measurements were carried out. We obtained a stability of $1.5 \times 10^{-10}$ at $1 \mathrm{~s}$. This result demonstrates that the objective short-term frequency stability of MAC-TFC, $6 \times 10^{-10}$ at $1 \mathrm{~s}$ can be obtained with our Cs-vapor cells.

Furthermore, a set of accelerated aging tests were performed with the micro Cs-vapor cells at temperatures a few tens degrees higher then the MAC operation temperature to examine the tolerance of the cells for long-term operation of the MAC. Figure 8 shows optical absorption spectrums obtained from a Cs-vapor cell before and after an aging test at $105^{\circ} \mathrm{C}$ for 2256 hours. The absorption spectrum of the cell after the aging is almost completely overlapped with that of the same cell measured before the test. This result clearly indicates that both $\mathrm{Cs}$ and a buffer gas remained in the cell without any significant changes in the pressure. The series of the aging tests and optical spectroscopic characterization allow us to estimate the lifetime of the cell to be at least $26048 \mathrm{~h}(\sim 3$ years $)$ at around the MAC operating temperature $\left(75^{\circ} \mathrm{C}\right)$.

Further investigation on the aging behavior of the cell was carried out using CPT spectroscopy. For this aging test, the cell was kept at $125^{\circ} \mathrm{C}$ for more than 3 weeks except for the time of CPT measurements performed every 2-3days. The variation in the frequency was within $3 \mathrm{~Hz}$ over 3 weeks. From this value, we estimate that the buffer gas pressure variation is $2.91 \times 10^{-5} \mathrm{kPa}$ for 1 day. If we assume the aging theory that the rate of aging doubles every $10^{\circ} \mathrm{C}$ of an increase in temperature, the equivalent pressure change per day at the operation temperature of MAC is $9.1 \times 10^{-7} \mathrm{kPa}$. To reach our objective frequency stability of $1 \times 10^{-11}$ at 1 day, the variation in the buffer gas pressure should be less than $1.84 \times 10^{-5} \mathrm{kPa}$. Thus, the result of this test is encouraging for the integration of the cell in our MAC.

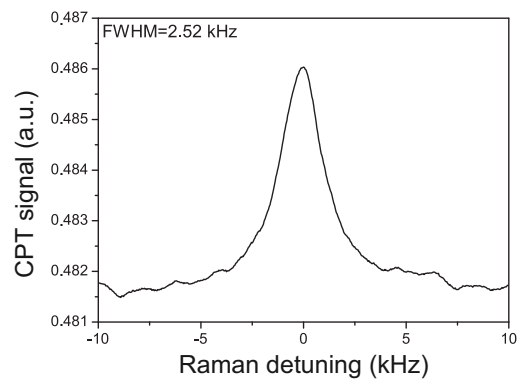

Figure 7: CPT resonance obtained in a Cs-vapor cell filled with $\mathrm{Ne}(8.1 \mathrm{kPa})$.

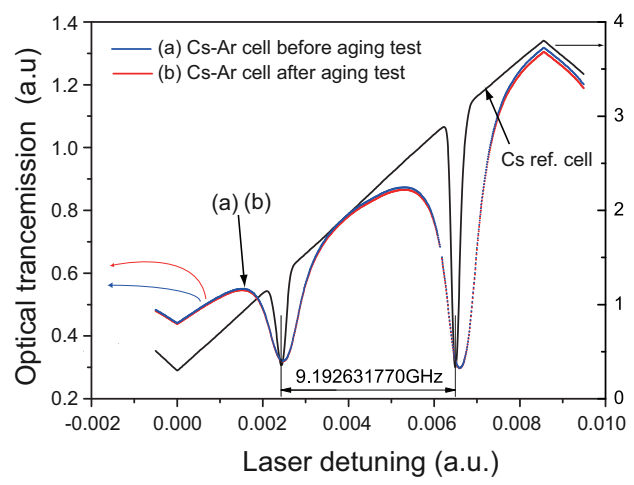

Figure 8: Optical absorption spectroscopy of micro $\mathrm{Cs}$-vapor cell (a) before and (b) after the aging test at $105^{\circ} \mathrm{C}$ for $2256 \mathrm{hr}$.

\section{THERMAL ANALYSIS OF CS-VAPOR CELL IN LTCC PHYSICS PACKAGE}

In order to package the MACs, we use the Low temperature co-fired ceramic (LTCC) technology. LTCC is a well-established multi-layer technology in the microelectronics packaging industry. Each of the layers is processed in parallel and only brought together in an accurately aligned stack immediately prior to firing. LTCC features the ability to embed various passive components (optical and electronic) into the ceramic package along with the cell, minimising the size of the completed module. One important requirement of the MACs is to control the temperature of their Cs-vapor cells to avoid frequency drifts. The cell temperature has to remain stable at an operating temperature $\left(75^{\circ} \mathrm{C}\right)$ although the MAC's surrounding-temperature can vary from $0^{\circ} \mathrm{C}$ to $55^{\circ} \mathrm{C}$. Thus, 
various packaging structures have been studied in order to reduce the temperature sensitivity of Cs-vapour cells with respect to change in ambient temperature. This sensitivity reduction is aimed to stabilize the cell temperature and to lower the power consumption._In this framework, thermal management of fully packaged Cs-vapour cell takes into account three heat transfer processes, namely conduction through physical parts, convection between central temperatures controlled part and enclosure, and radiation from the heated cell. To reduce these three processes, the LTCC frame, hanging the cell and containing heaters, is firstly patterned so that bridges connect its central area to the surrounding LTCC walls. These bridges create a high resistive path between the cell and its surrounding reducing the heat wastage by means of conduction. Then, to overcome heat wastage from conducto-convection process, complete physics package is enclosed in vacuum. Finally, radiations are decreased by metal coatings deposited onto LTTC surfaces surrounding the cell. Considering these solutions, we have performed thermal analysis by finite element method and found that the temperature sensitivity of such structure, with change in ambient temperature, is $<30 \mathrm{mK} / \mathrm{K}$. Moreover, at for instance $0^{\circ} \mathrm{C}$, the maximum temperature variation across the cell part (surrounding $\mathrm{Cs}$ atoms) is 55 $\mathrm{mK}$ (Fig. 9b). Thus, the power consumption to maintain the cell-temperature at its operating temperature $\left(75^{\circ} \mathrm{C}\right)$ is in the order of $<50 \mathrm{~mW}$ when the ambient temperature reaches $0^{\circ} \mathrm{C}$.

\section{CONCLUSIONS}

We investigated the fabrication and characterization of the Cs-vapor cell filled with a buffer gas as well as the thermal analysis of the packaged cell for thermal management of the MAC.

Cell-cavities were fabricated by the DRIE process optimized for very thick Si through-etching and subsequent $\mathrm{KOH}$ etching process to improve the surface quality of the DRIE-etched cavity sidewalls. The Cs-vapor cells were successfully filled with a buffer gas ( $\mathrm{Ne}$ or $\mathrm{Ar}$ ) and sealed using a two-step anodic bonding. The optical and CPT spectroscopic studies demonstrate that the cell contained a required buffer gas together with $\mathrm{Cs}$ vapor. The results of aging tests combined with these spectroscopic methods revealed that the cell possesses the inner atmosphere stability acceptable for the MAC application.

Thermal analysis shows that the effects of the different thermal processes have to be reduced. Thus, suspensions made of LTCC, vacuum environment and proper metal coatings should be applied in order to maintain the cell temperature constant.

\section{ACKNOWLEDGEMENTS}

This research was carried as a part of MAC-TFC project (EU FP7, grant no. 224132). (a)

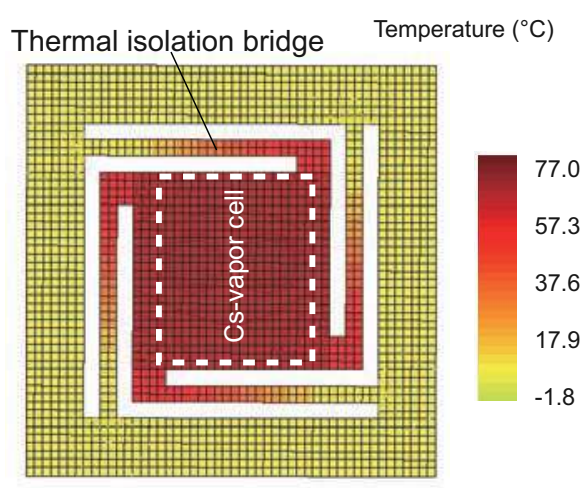

(b)

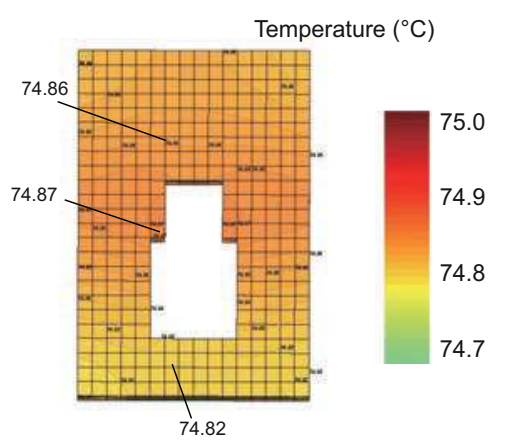

Figure 9: Temperature variation in (a) four-bridge suspension structure of LTCC package and (b) packaged cell for heater/sensor temperature of $77^{\circ} \mathrm{C}$ (cell-temperature of $\sim 75^{\circ} \mathrm{C}$ ) and ambient temperature of $0^{\circ} \mathrm{C}$.

\section{REFERENCES}

[1] S. Knappe, L. Liew, V. Shah, P. Schwindt, J. Moreland, L. Hollberg, J. Kitching, "A microfabricated atomic clock”, Appl. Phys. Lett., vol. 85, pp. 1460-1462, 2004.

[2] D. Younger, L. Lust, D. Carlson, S. Lu, L. Forner, H. Chanhvongsak, T. Stark, "A manufacturable chip-scale atomic clock", in proc. Transducers \& Eurosensors 2007, Lyon, June 10-14, 2007, pp. 39-44.

[3] R. Lutwak, A. Rashed, M. Varghese, G. Tepolt, J. Leblanc, M. Mescher, D.K. Serkland, K.M. Geib, G.M. Peake, "CSAC - the chip-scale atomic clock", in Proc. 7th Symposium on Frequency Standards and Metrology, Pacific Grove, October 5-11, 2008, pp. 452-462.

[4] J. Dziuban, C. Gorecki, L. Nieradko, V. Giordano, H. Maillotte, "Procédé de fabrication d'une cellule a métal alcalin pour micro-horloges atomiques", French Patent, DI 00619-01, October, 2006.

[5] L. Nieradko, C. Gorecki, A. Douahi, V. Giordano, J.C. Beugnot, J. Dziuban, M. Moraja, "New approach of fabrication and dispensing of micromachined cesium vapor cell", J. Micro-Nanolithogr. MEMS MOEMS, vol. 7, 033013, 2008.

[6] N. St. J. Braithwaite, "Introduction to gas discharges", Plasma Sources Sci. Technol., vol. 9, pp. 517-527, 2000.

[7] E. Bernabeu, J. M. Alvarez, "Shift and broadening of hyperfine components of the first doublet of cesium perturbed by foreign gases", Phys. Rev. A, vol. 22, pp. 2690-2695, 1980. 